\title{
De modelos internacionales y adaptaciones locales. El proceso de movilidad de la política de distritos económicos de la ciudad de Buenos Aires (2008-2019)
}

\section{About international models and local adjustments. The mobility process of the economic district's policy of the city of Buenos Aires (2008-2019)}

Carolina Gonzalez Redondo (1)

\begin{abstract}
RESUMEN
El artículo analiza el surgimiento y la formulación de la política de distritos en el sur de la ciudad de Buenos Aires, desde el año 2008. Para ello, recupera el abordaje de la movilidad de políticas atendiendo a su dimensión relacional y territorial. Con una estrategia metodológica cualitativa, desarrolla un dispositivo para el análisis de los discursos que permite advertir cómo esta política combina elementos de distintos modelos de gran circulación a nivel internacional, como los modelos de aglomeración y las economías y ciudades creativas. Luego, a partir del análisis de trayectorias de funcionarios y agentes públicos, da cuenta de la dimensión territorial de este proceso de movilidad, su carácter fijo y enraizado en el lugar. De este modo, el artículo invita a reflexionar sobre la relación entre procesos globales y locales, advirtiendo cómo los actores locales movilizan e introducen ideas que hacen posible el enraizamiento de políticas y modelos que circulan globalmente. Asimismo, propone un abordaje metodológico -el análisis discursivo- para estudiar la dimensión relacional de los procesos de movilidad.
\end{abstract}

Palabras clave: movilidad de políticas, distritos económicos, ciudades creativas, análisis del discurso.

\begin{abstract}
The paper analyzes the emergence and formulation of the district's policy in the south of the city of Buenos Aires, since 2008. For this purpose, it displays the policy mobility approach, taking into account its relational and territorial dimension. From a qualitative methodological strategy, it develops a procedure for the analysis of discourses that allows to see how this policy combines elements from different globally circulating urban models, such as clusters and creative cities. Then, from the analysis of the trajectories of public agents, it sheds light on the territorial dimension of this mobility process, on its fixed and embedded in place character. In this way, the article invites us to reflect on the relationship between global and local processes, noting how local actors mobilize ideas, models and policies circulating in relational geographies. Likewise, it proposes a methodological approach -discourse analysis- to study the relational dimension of mobility processes.
\end{abstract}

Keywords: policy mobility, clusters, creative cities, discourse analysis. 
La política de distritos, implementada desde 2008 por el Gobierno de la Ciudad de Buenos Aires (GCBA), combina elementos de diferentes modelos y herramientas de gestión urbana de gran difusión, como son las políticas de ordenamiento territorial basadas en la promoción de economías de aglomeración ${ }^{2}$, específicamente el modelo de cluster (Porter, 1995; 1998) y las propuestas de economías y ciudades creativas (Caravaca et al., 2013; Frost-Kumpf, 1998; Hyslop, 2012; Lazzeretti, 2008). El artículo analiza la circulación de estos modelos en el escenario local y se pregunta: ¿cómo llegaron estos modelos al ámbito de la ciudad de Buenos Aires?; ¿qué actores intervinieron en la formulación de esta política? ¿Se trata de un proceso de importación-exportación, con actores, escenarios y circunstancias específicas? $\mathrm{O}$, por lo contrario, ¿constituye un proceso de diseminación y circulación, que remite a procesos más difusos e inciertos? (Novick, 2009; Porto de Oliveira \& Pimenta de Faria, 2017). A partir de estos interrogantes, buscamos dar cuenta de la articulación de procesos globales y locales en la definición e implementación de políticas urbanas y, con ellas, en la producción de la ciudad.

Recuperamos el abordaje de la movilidad de políticas (policy mobility) (Hoyt, 2006; Mccann, 2011; McCann \& Ward, 2010; Peck \& Theodore, 2010; Temenos \& McCann, 2012), que concibe la circulación de políticas como procesos complejos, no lineales, dinámicos y desiguales, a través de los cuales las políticas mutan durante su viaje, adquiriendo formas diversas en cada contexto local. Esto es así porque la circulación no se produce en un plano inerte y sin forma, sino en un espacio que tiene la capacidad de moldear aquellos procesos que tienen lugar en él (Santos, 1996). Retomando un abordaje relacional y territorial (McCann \& Ward, 2010), entendemos que la formulación de políticas urbanas debe comprenderse en dos sentidos: en su dinamismo y relación con los circuitos globales de conocimiento, producidos en una geografía relacional focalizada en redes y flujos y, a la vez, en su carácter fijo y enraizado en el lugar, dado que las políticas son también intensa y fundamentalmente locales y territoriales (McCann \& Ward, 2010). La tensión entre relacional y dinámica y fija y territorial es una tensión productiva y necesaria que, como veremos, produce política y lugares (McCann \& Ward, 2010).

Para responder nuestros interrogantes, primero, desplegamos un análisis discursivo que permite identificar los supuestos en los que se basa esta política y así dar cuenta de su vínculo con los modelos de circulación internacional, focalizando en la dimensión relacional de la movilidad. Luego, analizamos las condiciones locales para el surgimiento de los distritos, fundamentalmente el tecnológico, atendiendo a la dimensión territorial. En las conclusiones, sintetizamos los principales aportes que produce el trabajo al campo de los estudios urbanos y, más específicamente, de la movilidad de políticas.

\section{Recorte espacio-temporal}

Los distritos económicos constituyen áreas de la ciudad de Buenos Aires en las que el gobierno local promueve la radicación de empresas e instituciones de un mismo sector económico. Actualmente, existen cinco distritos (Figura 1): Tecnológico (DT, 2008), Audiovisual (DAu, 2011), de las Artes (DA, 2012), del Diseño (DDi, 2013), y del Deporte (DDe, 2014). Con excepción del DAu, todos se emplazan en la zona sur de la ciudad y son presentados por el GCBA como una nueva propuesta para desarrollar estas "áreas deterioradas" de la ciudad y, a la vez, como una oportunidad para mejorar la inserción de Buenos Aires en la economía global. 
Para la atracción de estas actividades económicas, el gobierno local ofrece incentivos tributarios, financieros e inmobiliarios; despliega estrategias para la articulación con los actores empresariales promovidos; y realiza una fuerte inversión pública orientada a la renovación urbana de estas zonas. Así, los distritos constituyen una política paraguas que, combinando objetivos de desarrollo económico y urbano, profundiza y extiende el proceso de renovación urbana desplegado en el sur de la ciudad desde la década del ' 90 . Por cuestiones de espacio, a la hora de analizar la dimensión territorial del proceso de movilidad de esta política, en este artículo focalizamos en el surgimiento y definición del distrito tecnológico, el primero en ser creado, que-como se trabajará en otros artículos- luego se convierte en un modelo de circulación a nivel local.

Figura $\mathrm{N}^{\circ} 1$.

Ubicación de los distritos

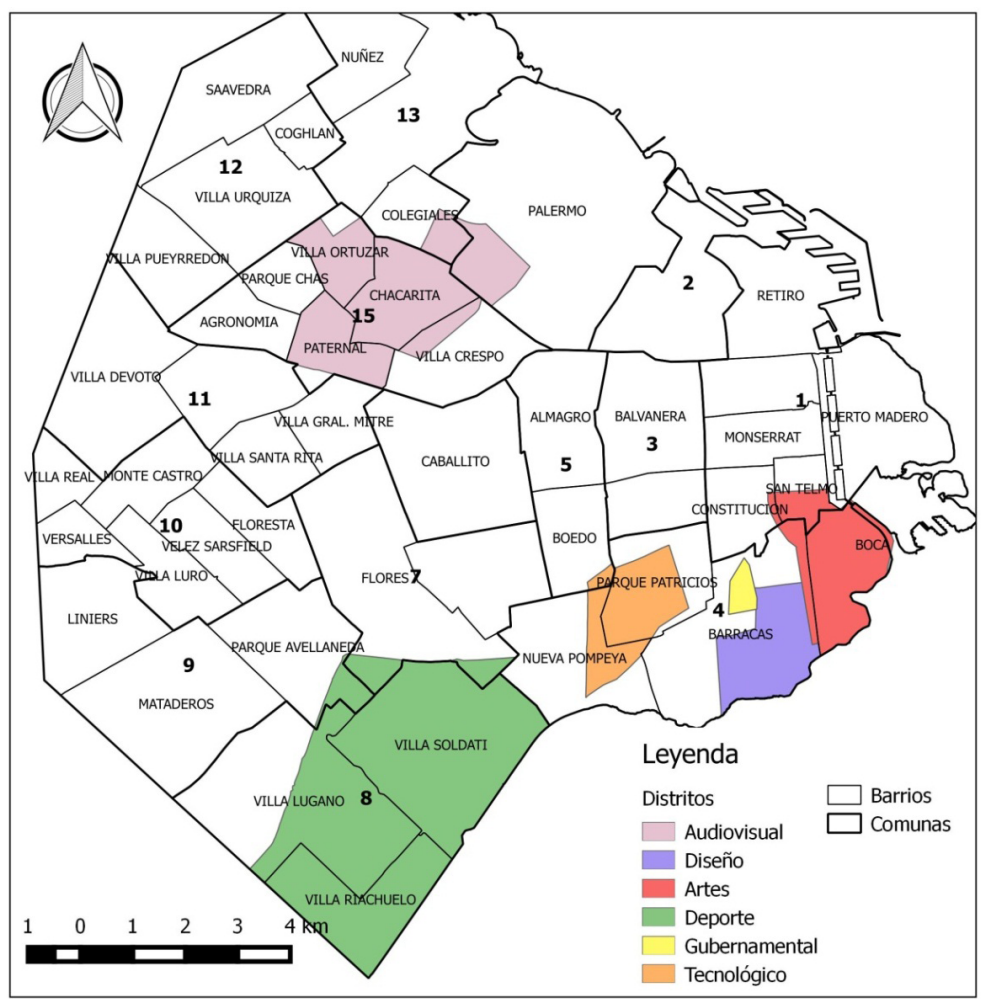

Fuente: elaboración propia en base a información del GCBA.

\section{Consideraciones metodológicas}

Diseñamos una estrategia metodológica cualitativa, con orientación empírica, descriptiva e interpretativa. Para la producción de evidencia empírica, combinamos diversas fuentes y técnicas de recolección de datos, tales como documentos vinculados a la política (marcos normativos, partidas presupuestarias, gacetillas de prensa y materiales gráficos); y entrevistas semi-estructu- 
radas a actores involucrados en la política (funcionarios públicos, agentes del GCBA, empresarios, agentes del sector inmobiliario) ${ }^{3}$.

Para su abordaje, combinamos el análisis interpretativo con un dispositivo para el análisis de un corpus heterogéneo de discursos ${ }^{4}$ que, retomando la perspectiva teórica de la escuela francesa, combina elementos de las teorías de la enunciación y la argumentación ${ }^{5}$. El análisis de recursos lingüísticos y operaciones enunciativas permite identificar cómo se construye a sí mismo el enunciador -con qué ethos ${ }^{6}$ se asocia-y qué enunciatario configura. En tanto, el análisis de las estrategias argumentativas, el despliegue de topos retóricos y lugares comunes (Amossy, 2000), ayuda a identificar las construcciones argumentativas y tematizaciones puestas en juego en el entramado discursivo de la política. Nuestro análisis se asienta en el nivel intradiscursivo, ese plano en el que se sitúan las operaciones enunciativas y argumentativas, donde el sujeto-enunciador aparece administrando la polifonía (Montero, 2014). Sin embargo, tiene como horizonte su vínculo con el interdiscurso, ese nivel constitutivo en el que el sujeto es hablado por el discurso, es un sujeto del inconsciente atrapado por las formaciones discursivas e ideológicas?

\section{De los modelos de aglomeración y economías creativas a los distritos económicos}

Al mencionar algunos referentes, en el entramado discursivo de la política se asume explícitamente el vínculo de los distritos con iniciativas similares llevadas a cabo "en distintas ciudades de los cinco continentes" (Revista GigaBA, 2013: 45). Según el ex Ministro de Desarrollo Económico y promotor de esta política, Francisco Cabrera,

Un distrito es pensar la ciudad como espacios temáticos en los que tiene lugar el desarrollo de una industria de alto valor y la mejora de un barrio postergado a partir de la sinergia público-privada (Cabrera, 2012).

Esta definición ilustra cómo en los discursos sobre los distritos se recuperan supuestos que subyacen a los modelos sobre economías de aglomeración, industrias y ciudades creativas que circulan internacionalmente. Asimismo, de esta secuencia se desprende el doble objetivo de la política: la promoción de determinadas actividades económicas ("desarrollo de una industria de

\footnotetext{
También incorporamos entrevistas realizadas por colegas (Eugenia Goicoechea, Ivana Socoloff y Natalia Lerena) en el marco de otras investigaciones, a quienes agradecemos por su colaboración.

4 El corpus reúne discursos pertenecientes a diversos tipos y géneros discursivos, producidos en diferentes contextos espacio-temporales desde distintas instituciones. Incluye versiones taquigráficas de la Legislatura, notas de opinión escritas por funcionarios públicos, prólogos de libros y material de difusión sobre los distritos, fundamentos de proyectos de ley, boletines electrónicos del distrito tecnológico, y entrevistas a funcionarios y agentes públicos.

5 Esta perspectiva invita a pensar en la dimensión conflictiva de todo discurso dado que su sentido no es unívoco, existe una lucha por su fijación. No hay una "democracia discursiva", no cualquier voz es legítima en cualquier momento y lugar, sino que las tematizaciones responden a tendencias hegemónicas y leyes tácitas que regulan lo posible de ser dicho en un momento dado (Angenot, 1989).

${ }_{6}$ Remite al tono y al cuerpo que construye el enunciador a lo largo del enunciado. El ethos es el garante de la enunciación y su identificación permite advertir el posicionamiento del enunciador, el enunciatario que construye y los ámbitos institucionales que legitiman su enunciado, en asociación con una determinada formación discursiva (Maingueneau, 1996).

Acuñado por Foucault (1969) y reformulado por Pecheux (1971), el concepto refiere a "lo posible de ser dicho desde una posición dada en una coyuntura dada" (Pecheux, 1971 citado en Maldidier, 1990:206).
} 
alto valor agregado") y el desarrollo de la zona sur de la ciudad ("la mejora de un barrio postergado"). Estos dos objetivos se presentan de manera conjunta a partir de una serie de construcciones argumentativas ${ }^{8}$ sobre los distritos, que tienen entre sus condiciones de producción (Verón, 1988) a los discursos sobre economías de aglomeración y ciudades creativas.

Una primera construcción argumentativa -que remite directamente a la definición de cluster de Porter $^{9}$ (1998)- es que la concentración geográfica de empresas e instituciones de un mismo sector económico genera intercambios que favorecen el crecimiento de toda la cadena, a través de las denominadas "sinergias":

La política de distritos [...] procura desarrollar diversas industrias creativas en un espacio geográfico acotado, para favorecer el intercambio entre sus miembros, fortalecer su crecimiento y estimular su desarrollo (GCBA, 2017: 4).

Este supuesto se articula con una segunda construcción argumentativa -referida al "efecto cluster"- que sostiene que la concentración de actividades económicas derrama en inversiones y empleo, motorizando de este modo procesos de desarrollo. La metáfora del derrame, de larga tradición en la teoría económica liberal, forma parte de las premisas sobre los clusters y es construida, en los discursos analizados, mediante un círculo virtuoso que sintetizamos así: a mayor cantidad de empresas, mayor cantidad de personas/consumidores, mayor consumo, mayor actividad comercial, mayor empleo, mayor inversión en espacio público y servicios. Veamos un ejemplo:

En la medida que se instala una empresa, se mejora la zona, la luminaria, la vereda, los tipos limpian, tienen seguridad, ese espacio lo transformás, lo embellecés, si querés. Y además, lo desarrollás económicamente porque traés gente que consume. (Entrevista a la Gerente Operativa de los Distritos Económicos, enero de 2019).

La metáfora del derrame permite combinar los objetivos de desarrollo económico y urbano y, de este modo, legitimar a los distritos como una política de desarrollo para "zonas postergadas", específicamente el sur de la ciudad. A su vez, ésta se apoya en una premisa -también de la teoría de clusters- que sostiene que las empresas requieren de ventajas competitivas para mejorar su productividad, cobrando importancia el denominado "clima de negocios" (Porter, 1995). Así, las inversiones en el entorno construido forman parte de la intervención pública orientada a crear el "clima de negocios", potenciando las ventajas competitivas de la ciudad, en general, y de los barrios de los distritos, en particular.

Bajo el paradigma de la competitividad territorial, la enumeración de ventajas competitivas constituye un argumento de venta de la ciudad, orientado a "posicionar" a Buenos Aires como una "marca-ciudad". Se establece una analogía entre la ciudad y la empresa que remite, por un lado, a la ciudad como mercancía (Vainer, 2000) -en tanto que las ventajas competitivas se convierten en atributos para su posicionamiento en el mercado- y, por el otro, a la ciudad como empresa (Vainer,

Refiere a los argumentos movilizados para legitimar, producir consenso y/o persuadir en torno a una posición.

Según Porter (1998: s/n), los cluster son "concentraciones geográficas de empresas e instituciones interconectadas en un campo determinado [...] [que] derraman río abajo hacia canales y clientes y posteriormente hacia productores de productos complementarios y hacia empresas de industrias relacionadas por habilidades, tecnologías o insumos comunes". 
2000), dado que la enumeración de estas ventajas se produce recuperando lexemas propios de la jerga empresarial, como "éxito", "marca", "diferenciación", remitiéndonos a formaciones discursivas ligadas al marketing y los negocios.

Las ventajas competitivas referidas en nuestro corpus remiten a los discursos sobre economías y ciudades creativas (Florida, 2002; 2007; Lazzeretti, 2008; Frost-Kumpf, 1998; Hyslop, 2012). Particularmente, identificamos una construcción argumentativa que postula la creatividad y el talento como ventajas competitivas asociadas al "ser porteño":

Cuando pensamos el posicionamiento de Buenos Aires, y tenemos una mirada holística de la ciudad, indefectiblemente las dos cualidades que resaltan son la creatividad y el talento. Por tanto, a la hora de pensar políticas que desarrollen estas cualidades y sustenten una marca-ciudad que diferencia a Buenos Aires, coincidimos en una estrategia: fomentar aquellas industrias intensivas en conocimiento y que emplean alto valor agregado. Las industrias creativas, las del talento: la tecnología, la industria audiovisual, el arte y el diseño (Cabrera, 2012:24).

En esta secuencia, ambos atributos son presentados como cualidades intrínsecas al "ser porteño" y, mediante un efecto metonímico, como ventajas competitivas de la ciudad de Buenos Aires. En sintonía con los enfoques que destacan los factores "blandos" del desarrollo (ver Cuervo González, 2017; Riffo, 2013), identificamos otra construcción argumentativa que postula como un imperativo la valorización de recursos intangibles, como la creatividad y el talento. Así, las industrias creativas son tematizadas como las actividades del futuro, asociadas a lexemas como "modernas" e "innovadoras", y en oposición a las industrias culturales, que "remiten a cosas del pasado". En estos discursos, el rol del Estado consiste en transformar lo cultural y artístico en "creativo", y a los artistas en "empresarios sustentables y competitivos" (Cabrera, 2012:24). La referencia a la competitividad se articula con una construcción argumentativa -que está en el centro de los modelos sobre ciudades creativas- que tematiza a estas industrias como generadoras de "empleo de alto valor agregado. Esto aparece como una idea fuerza que legitima la promoción de estas actividades, en la medida en que aportan al interés común, como se desprende de la siguiente secuencia:

[...] no habrá ciudad creativa si no enfrentamos seriamente el desafío de generar oportunidades reales para que no haya más pobreza en nuestro país. Las industrias creativas ofrecen la posibilidad de generar empleo de alto valor agregado y dinamizan, asimismo, todo el entramado productivo, Tenemos una oportunidad única entre manos y debemos aprovecharla. (Avogadro, 2012:30)

Aquí, se tematiza a los distritos en términos prescriptivos ("tenemos qué", "debemos") y, así, se refuerza que el Estado "debe" promocionar las industrias creativas en tanto que constituyen una "oportunidad" para acabar con la pobreza. Éstas se presentan como aquellas "actividades del futuro" que reemplazan las industrias tradicionales, generan empleo y son adecuadas para "revitalizar" y "renovar" zonas con un pasado industrial; argumento que remite a los enfoques que postulan a la creatividad como fuerza motor del desarrollo urbano. 
Los distritos en el sur de la ciudad son presentados como una iniciativa para favorecer el desarrollo económico y urbano de estos barrios. Ahora bien, identificamos diversas tematizaciones sobre el "desarrollo", que asociamos a los distintos ethos construidos en cada discurso (Maingueneau, 1996) y que, a su vez, se relacionan con los diferentes sujetos que -parafraseando a Bohoslavsky \& Soprano (2008)- habitan y dan vida a la política de distritos. Si bien el ethos refiere al enunciador y no debe confundirse con el sujeto-locutor, es posible observar cierta correspondencia entre el enunciador construido, su presentación de sí, y el sujeto que le da cuerpo, su trayectoria personal y profesional.

En aquellos discursos con un enunciador asociado a un ethos que combina el rol de funcionario público y de empresario, el significante "desarrollo" no aparece como tal, sino que predomina una construcción argumentativa que privilegia la inserción de Buenos Aires en la economía mundial. Así, tanto los distritos como la "promoción de industrias estratégicas" son presentados como una herramienta para "recomponer el lugar de Buenos Aires en el mundo", "ubicar a la ciudad en el mapa de los inversores o clientes". Los sujetos-locutores de estos enunciados son aquellos funcionarios del área de Desarrollo Económico, e incluso el propio ex Jefe de Gobierno, que -como veremos a continuación- tienen una amplia trayectoria profesional en el mundo empresarial.

En otros discursos, con un ethos de experto que utiliza un vocabulario técnico con relación a la planificación de la ciudad, los distritos aparecen tematizados como una propuesta para "desarrollar la zona sur" de Buenos Aires. Prima un diagnóstico que refiere a "diferencias territoriales sustanciales" que se expresan en precios del suelo más bajos en el sur de la ciudad. Aquí aparece explícitamente el lexema "desarrollo" asociado a los procesos de "mejora en la calidad de vida de los habitantes" y de valorización del suelo en las zonas de intervención. Los sujetos-locutores de estos enunciados son funcionarios públicos del Ministerio de Desarrollo Urbano, con trayectoria profesional en el ámbito privado, pero también técnicos o funcionarios de carrera de dicha cartera. Estos discursos tienen entre sus condiciones de producción (Verón, 1988) otros discursos en torno al deterioro del sur y su desequilibrio con respecto al norte, cuestión (Oszlak \& O'Donnell, 1981) que es problematizada como tal desde comienzos del siglo XX (González Redondo, 2020).

Finalmente, en los documentos del GCBA que buscan presentar la política de distritos a legisladores y ciudadanos, el "desarrollo" aparece tematizado en un triple sentido: económico, urbano y social. Lo que actúa como nexo entre estos objetivos es, precisamente, la metáfora del derrame. La radicación de empresas supone desarrollo económico y, "a su par", el desarrollo urbano y social del área:

[se propone el distrito tecnológico] para que aún manteniendo su fisonomía industrial, se concentren actividades de base tecnológica, innovadoras e intensivas en el uso del conocimiento, estimulando a la par su desarrollo urbanístico, comercial y social (LCABS, 2008).

Resulta ilustrativo cómo se presentan los objetivos del distrito tecnológico en los fundamentos del proyecto de ley. Allí, se mencionan las características del distrito en tres párrafos con el siguiente orden: "de renovación urbana", "de desarrollo económico", "de desarrollo social", unificando los tres componentes del proyecto. La "renovación urbana" se asocia a la revalorización de la zona. El "desarrollo económico" remite a una "oportunidad para reconvertir a Buenos Aires en un eje internacional competitivo generador de tecnología y conocimiento". El tercer componente 
unifica los otros dos, bajo la premisa de que el sector tecnológico "brinda oportunidades laborales y de inclusión para toda la sociedad". De hecho, la radicación de empresas, la cantidad de metros cuadrados construidos, los empleos trasladados y el monto invertido son los indicadores del "éxito" de la política, presentándose como los efectos del "desarrollo".

Estos indicadores se apoyan en el supuesto del derrame que permite asociar todos estos elementos con un desarrollo económico, urbano y social. Entre estos indicadores identificamos un énfasis en el desarrollo inmobiliario (expresado como "metros cuadrados construidos") que se aleja parcialmente de los presupuestos de los modelos de aglomeración y las políticas creativas. Este impulso al desarrollo y la valorización inmobiliaria responde -como veremos a continuacióna los arreglos políticos locales de la movilidad de políticas (the local politics of policy mobility) (Temenos \& McCann, 2012).

\section{Las condiciones locales de la movilidad de políticas urbanas}

En este apartado, nos centramos en la dimensión territorial del proceso de movilidad de esta política, focalizando en el distrito tecnológico. Así, nos preguntamos cómo y por qué se crea este distrito y qué actores estuvieron involucrados en su formulación. Combinamos la propuesta teórica sobre movilidad de políticas con la tradición de la sociología política (Bohoslavsky \& Soprano, 2008; Gené, 2011; Giorgi, 2014; Morresi \& Vommaro, 2011; Vommaro \& Gené, 2017), que se pregunta por el funcionamiento efectivo de la política en minúscula (Vommaro \& Gené, 2017). Esto es, por las negociaciones y decisiones movilizadas por el entramado de actores e intereses que actúan el Estado. Desde esta perspectiva, el origen socio-cultural, las redes de sociabilidad, las trayectorias biográficas y profesionales -o los mundos sociales de pertenencia (Vommaro, 2017)- de los agentes estatales influyen en su quehacer político, en sus repertorios de acción y sus modos de acceder a la política. Como veremos, las trayectorias de los agentes públicos inciden también en las características que asume una política urbana. De ahí que personalicemos el Estado (Bohoslavsky \& Soprano, 2008) y nos preguntemos por los sujetos que habitan y dan vida a la política de distritos.

Dos ex funcionarios del Ministerio de Desarrollo Económico (MDE) son reconocidos como los "padres" de los distritos económicos: el ex ministro Francisco Cabrera y el ex Subsecretario de Inversiones, Carlos Pirovano. Según este último, el distrito tecnológico surge a raíz de una estrategia de desarrollo económico de la Subsecretaría de Inversiones, creada por la gestión de $\mathrm{PRO}^{10}$ dentro del MDE. En este marco, se definieron las "industrias estratégicas" de la ciudad de Buenos Aires a partir de identificar sus "ventajas competitivas" y se creó un departamento de atracción de inversiones, actualmente denominado InvestBA. Entre las actividades definidas como estratégicas -Tecnología, Información y Comunicación (TIC), inmobiliarias, turismo y audiovisual (GCBA, 2009)- se comenzó con la promoción de las actividades TIC, por ser el sector más consolidado y formalizado:

- Propuesta Republicana (PRO) es el partido político que gobierna la ciudad de Buenos Aires desde 2007. Mauricio Macri fue jefe de gobierno entre 2007 y 2015. Lo sucedió Horacio Rodriguez Larreta, desde 2015 a la actualidad. 
Dijimos vamos a hacer leyes de promoción de cada una de las industrias estratégicas. Empezamos de la más fácil a la más difícil. La más fácil era la tecnológica. ¿Por qué era la más fácil? Porque había experiencia internacional, porque son industrias que existen. (...) del entorno de las empresas creativas son las que tenían la cadena de valor más consolidada (Entrevista al ex Subsecretario de Inversiones del MDE, mayo de 2015).

Desde principios de milenio, la actividad tecnológica es crecientemente promovida por instituciones públicas nacionales y locales, lo que se manifiesta en la creación de polos tecnológicos en distintas ciudades del país (Finquelievich, 2018). Así las propuestas de fomento a las economías creativas para atraer inversiones, así como las iniciativas de articulación público-privada para desarrollar "áreas deterioradas" no son exclusivas de esta gestión de gobierno ni de esta política en particular. Por lo contrario, se reconocen antecedentes similares -aunque no igualesen los primeros proyectos de polos motorizados por la Corporación Sur (Arqueros, 2017; Socoloff et al., 2012). Estas continuidades se explican por los procesos de movilidad más difusos, en los que las ideas viajan como formaciones discursivas que marcan lo posible de ser pensado y hecho en un momento determinado, legitimando y condicionando las formas de concebir la ciudad, el desarrollo y las herramientas de gestión urbana.

Sin embargo, la particularidad del DT es la promoción de la actividad tecnológica en un sector acotado de la ciudad. Esta decisión surgió como producto del intercambio entre empresarios del sector TIC -algunos de los cuales habían participado de proyectos previos- y funcionarios del gobierno local. Los requisitos de la industria eran tres: infraestructura y conectividad, recursos humanos calificados y "metro cuadrado barato". Del análisis de estas necesidades nació la propuesta del distrito tecnológico:

(...) de ninguna manera estábamos pensando en distritos, estábamos pensando en promover las actividades relacionadas con la tecnología de la información y la comunicación. Pero, qué pasa, cuando nos juntamos con estas empresas, nos plantean ciertas necesidades. Primero, recursos humanos, el software es una industria que tiene déficit de recursos humanos. Después nos dicen, lo segundo es el metro cuadrado. Pero estos metros cuadrados tienen que tener infraestructura, redundancia de electricidad, redundancia de fibra óptica, tienen que tener ciertas características. (Intervención del ex Subsecretario de Inversiones del MDE, en un encuentro con agentes del sector inmobiliario. Noviembre de 2013)

Entonces dijimos no vamos a hacer mil kilómetros de fibra óptica, pero hacerlo en un lugar chiquitito no es caro. (...) Ahí apareció la idea de hacer un distrito, de hacer un cluster. Además la idea de la clusterización, el tema de la economía de aglomeración. Después empezó a venir todo lo que venía después. Lo primero es cómo podemos hacerlo bueno, bonito y barato. (Entrevista al ex Subsecretario de Inversiones del MDE, mayo de 2015)

Así, el distrito tecnológico surge, inicialmente, como respuesta a una cuestión (Oszlak \& O'Donnell, 1981) vinculada a la necesidad de fortalecer económicamente a la industria TIC. De la articulación con empresarios TIC, en su proceso de diseño, esta política adopta la forma de cluster. Es recién entonces que se recurre a modelos, discursos e ideas de referencia que permiten legitimar localmente una política que responde a necesidades e intereses de determinados actores, en este caso, las empresas TIC (González, 2011; McCann, 2011; Temenos \& McCann, 2012). 
Una vez definido el cluster, se evaluaron sus incentivos y su localización. Dos cuestiones diferencian la nueva propuesta de sus antecesoras: a) la idea de un distrito abierto, vinculado al entorno barrial, a diferencia de los polos cerrados proyectados durante los años 2000, y b) la cuestión de la seguridad jurídica, entendida como un elemento clave para la atracción de inversiones y la generación del "clima de negocios" (Porter, 1998). Así, cada distrito fue creado mediante una ley que regula sus beneficios y beneficiarios por un plazo de 10 a 20 años, incorporándolos al marco legal de la ciudad de Buenos Aires.

Ahora bien, la elaboración de un proyecto de ley y su posterior aprobación requiere estrategias de consenso, primero, entre los distintos organismos del gobierno local y, posteriormente, entre los diversos bloques que conforman la Legislatura. Este proceso implicó generar acuerdos con la Administración Gubernamental de Ingresos Públicos (AGIP), organismo autárquico encargado del proceso recaudatorio de la ciudad, en relación a los incentivos fiscales, sus plazos y controles. Asimismo, fue necesario involucrar a las áreas del Ministerio de Desarrollo Urbano (MDU) a cargo de la planificación urbana de la ciudad, para definir el polígono en el que se ofrecerían los beneficios.

Considerando los requisitos de los empresarios TIC, el MDE propuso tres posibles ubicaciones para el distrito tecnológico: la zona de la Av. Ing. Huergo, al norte de La Boca y próxima a Puerto Madero; sobre Av. Patricios entre La Boca y Barracas; y el área de Av. 9 de Julio Sur, cerca del Centro Metropolitano del Diseñon:

[...] tres zonas que a mí me gustaban porque me hacían el trabajo fácil. Yo te digo la verdad, no me implicaba un esfuerzo territorial demasiado... Vos pensá que mi objetivo no era desarrollar un barrio, mi objetivo era promover una industria [...] Cuando hacemos la presentación en Desarrollo Urbano, Héctor Lostri'12 se para y dice "yo propongo Parque Patricios" (Entrevista al ex Subsecretario de Inversiones del MDE, mayo de 2015).

Así, en la formulación del distrito tecnológico también intervino la Subsecretaría de Planificación del MDU, un área que se había trazado como objetivo el desarrollo del sur de la ciudad. Para esta área de gobierno, el distrito tecnológico encaja perfectamente como una propuesta para generar tejidos mixtos, repoblando las instalaciones que quedaron en desuso por el proceso de desindustrialización parcial de esta zona de la ciudad. En este aspecto fue central la presencia de un funcionario de carrera del GCBA que venía analizando la zona sur y sus posibilidades de reconversión industrial (ver Álvarez de Celis, 1999; 2000; 2007) ${ }^{13}$. De este modo, convergen los objetivos de ambas carteras, mediante un proyecto que busca tornar atractivas para la inversión a estas zonas de la ciudad:

Así que nos juntamos, nos pusimos a charlar y surgió una estrategia que es claramente [...] la confluencia de ese enfoque económico más ese enfoque territorial, que es lo que

Finalmente estas primeras áreas preseleccionadas fueron parte del distrito de las artes y/o del diseño.

Héctor Lostri era el Subsecretario de Planeamiento Urbano, dentro del Ministerio de Desarrollo Urbano del GCBA.

Fernando Álvarez de Celis es Licenciado en Geografía por la Universidad de Buenos Aires y Magister en Economía Urbana por la Universidad Di Tella. Como agente de perfil técnico, se desempeñó en distintas áreas del GCBA desde 2001. Con la gestión de PRO, ocupó cargos de mayor jerarquía y luego, con el triunfo de Cambiemos en las elecciones nacionales de 2015, pasó a desempeñarse como funcionario público en la Administración Pública Nacional. 
nosotros ya hemos Ilamado [...] la estrategia distritos (Intervención del ex Subsecretario de Inversiones del MDE en la Expo Real Estate de 2011)

Esta convergencia de objetivos de desarrollo económico y urbano es presentada como una fortaleza, como producto del consenso entre distintos saberes expertos y miradas disciplinarias, el "enfoque económico" y el "enfoque territorial", aunque la mirada urbana quede subsumida a la económica. Los incentivos y localización del distrito tecnológico derivan de esta convergencia de objetivos. De hecho, los límites del polígono de intervención se definieron teniendo en cuenta, las necesidades de la industria TIC (que requiere de los servicios y equipamientos urbanos disponibles) y del sector inmobiliario, que demanda áreas con una baja incidencia del suelo.

Los incentivos también tuvieron en cuenta a ambos sectores de la economía. Los beneficios fiscales -y específicamente el de ingresos brutos, que grava la actividad económica- sirven de estímulo para que las empresas TIC se instalen en el distrito. Esto se complementa con exenciones tributarias asociadas a la actividad inmobiliaria con el fin de incentivar el desarrollo de oficinas en la zona. El arribo de empresas tecnológicas demandó una tipología inmobiliaria hasta entonces inexistente en esa zona de la ciudad: los edificios de oficinas. Así, el incentivo al sector inmobiliario se produjo también de manera indirecta, estimulando la demanda.

Como vemos, las características de la ley del DT y su lugar de emplazamiento fueron consensuados por funcionarios del sector público junto con las empresas vinculadas a la actividad promocionada. Asimismo, las trayectorias personales y profesionales de estos funcionarios, especialmente Francisco Cabrera y Carlos Pirovano, también incidieron en la definición e implementación de la política. Ambos funcionarios tienen una extensa trayectoria laboral en el mundo corporativo ${ }^{14}$ (Goicoechea, 2016), lo que según otro funcionario del mismo ministerio, colaboró en las características que adoptó la política:

Creo que la experiencia en particular de Pancho y de Carlos en el mundo corporativo ayudó, siendo el primer distrito el distrito tecnológico, porque le hablaron de igual a igual a una serie de compañías que pensaban atraer (Entrevista al ex Subsecretario de Industrias Creativas del MDE, mayo de 2015).

En tanto, la vinculación con las premisas sobre ciudades creativas puede atribuirse a la participación de Enrique Avogadro, ex Subsecretario de Industrias Creativas, quien tiene un perfil orientado a las políticas culturales ${ }^{15}$. Los entonces funcionarios del MDU también una trayectoria

14 Francisco Cabrera es ingeniero en electricidad y electrónica por la Universidad de Mendoza y posee un Máster en Administración de Empresas de la Escuela Superior de Economía y Administración de Empresas (ESEADE). Carlos Pirovano es Licenciado en Economía por la Universidad de Buenos Aires. Ambos desarrollaron su carrera en el ámbito privado, principalmente en el holding financiero Grupo Roberts y en la administradora privada de jubilaciones y pensiones Máxima AFJP, empresa en la que Cabrera se desempeñó como CEO (Goicoechea, 2016). Cuando Mauricio Macri asumió como presidente en 2015, ambos funcionarios pasaron al ámbito nacional.

15 Enrique Avogadro estudió en el Colegio Cardenal Newman, al igual que Mauricio Macri y muchos referentes de PRO. Es Licenciado en Estudios Internacionales por la Universidad Torcuato Di Tella y estudió las maestrías de Administración y Políticas Públicas en la Universidad de San Andrés y de Gestión de Contenidos en la Universidad Austral. Ingresó a la gestión pública en 1998, y en 2008, se incorporó a PRO. Pasó por distintos cargos en el GCBA y, entre 2015 y 2017 fue funcionario del gobierno nacional. Luego de unos meses fuera de la gestión, en diciembre de 2017 asumió como Ministro de Cultura de la ciudad de Buenos Aires. 
en el mundo privado, pero ligada al sector inmobiliario ${ }^{16}$. Así, la estrecha articulación entre sector privado y público está dada también por las trayectorias personales y laborales de los funcionarios involucrados en su definición. Este vínculo cercano entre sector público y privado se revela, a su vez, en la existencia de un lenguaje compartido entre los funcionarios y los empresarios de las actividades promovidas. La participación de empresarios en organismos públicos de distinto nivel no es un fenómeno novedoso (Castellani, 2016; Menazzi, 2018). La diferencia, a partir de la gestión de PRO en CABA y de Cambiemos en Nación, es su magnitud, extensión y visibilidad (Castellani, 2016). Siguiendo a Vommaro (2017:43), el ámbito empresarial -junto con el del voluntariado- constituyen los mundos exteriores a la vida política que nutren de elementos al ethos político de PRO, entendido como "su repertorio dominante de prácticas, visiones del mundo y formas de dar cuenta de él en discursos y presentaciones públicas". Estos mundos sociales de pertenencia (Vommaro, 2017) -que refieren al background social, la posición de clase y los medios profesionales y redes de sociabilidad actualizadas de los miembros de un partido- constituyen anclajes sociales que proveen marcos de sentido, condicionamientos y recursos para la acción. No sólo permean la vida partidaria, su organización interna, sus rituales y valores morales, sino que -como vemos en este caso- condiciona la forma de concebir y hacer las políticas públicas. El lenguaje compartido con los empresarios (el "hablar de igual a igual"), el modo de presentar y legitimar discursivamente la política de distritos y los instrumentos implementados para medir su "éxito", e incluso las características que adopta la política en su proceso de implementación, especificamente en relación a las formas de articulación entre actores públicos y privados, remiten a esquemas, discursos, repertorios y estéticas propios el mundo corporativo.

La referencia a casos "exitosos" de otras partes del mundo -específicamente la experiencia del 22@ Barcelona17- contribuye a construir consensos en torno a la creación el distrito tecnológico. Esto es así porque los modelos movilizados por consultores suelen acompañarse de un "sello de excelencia y plus de prestigio", de un conjunto de supuestos incuestionables que legitiman, en este caso, al distrito tecnológico a nivel local (McCann, 2004; Temenos \& McCann, 2012). Así, las recurrentes referencias por parte de los funcionarios locales al caso del 22@ Barcelona constituye más una estrategia para la legitimación del distrito tecnológico a nivel local, que un proceso de transferencia y/o traducción (Novick, 2005; Porto de Oliveira \& Pimenta de Faria, 2017) llevado a cabo por actores vinculados al modelo de referencia. Por lo contrario, la tarea de diseño del DT, retomando algunas cuestiones del 22@, estuvo a cargo de los funcionarios locales, en colaboración con algunos asesores de universidades privadas ${ }^{18}$.

Asimismo, como analizamos en otros trabajos (González Redondo, 2019), el distrito tecnológico de Buenos Aires guarda más diferencias que semejanzas con este modelo. Las similitudes

\footnotetext{
Tanto Daniel Chaín (Ministro de Desarrollo Urbano, 2007-2015) como su mano derecha, Héctor Lostri (Secretario de Planeamiento, 2007-2015) son arquitectos por la UBA e hicieron la Maestría en Economía Urbana de la Universidad Di Tella. Ambos desarrollaron su carrera en el ámbito privado. Daniel Chaín fue ejecutivo del Grupo Socma, empresa de la familia Macri que se dedicaba a la construcción, como contratista del Estado local y nacional. Héctor Lostri formó parte del estudio de arquitectura 3S, cuyo socio fundador es Daniel Chaín, y, posteriormente, de Roosevelt Desarrollos Inmobiliarios (Arqueros, 2017).

17 El plan 22@ Barcelona del año 2000 promueve la transformación de 200 hectáreas industriales del área del Poblenou en un distrito productivo vinculado a las economías del conocimiento, mediante cambios en la normativa urbana y la proyección de inversión público-privada (ver Barcelo Roca, 2015).

18 Mediante un Convenio de Asistencia Técnica entre la Universidad Torcuato Di Tella y la Subsecretaría de Planeamiento Urbano del Gobierno de la Ciudad de Buenos Aires, se elaboró un informe sobre el distrito tecnológico (Sanguinetti \& Goytía, 2009). De todas formas, se trata de trabajos elaborados con posterioridad a la creación del distrito.
} 
se basan fundamentalmente en las construcciones argumentativas que se despliegan en ambos casos para legitimar estas políticas y en las premisas que operan como su trasfondo conceptual. Las diferencias, en tanto, se vinculan con los instrumentos, incentivos e instituciones que forman parte del diseño y la implementación de cada política; es decir con los arreglos políticos locales que habilita y permite cada ciudad. Así, el distrito tecnológico -con sus características y adaptaciones- tomó vida propia, manteniendo sólo una relación simbólica con su modelo de referencia (Peck \& Theodore, 2010) ${ }^{19}$.

\section{Conclusiones}

La política de distritos y, específicamente el distrito tecnológico, es el resultado de un proceso decisorio en el que intervinieron fundamentalmente actores locales (funcionarios públicos y empresarios). Sus características, beneficios y beneficiarios responden a la denominada política en minúscula (Gené, 2017), a los arreglos locales de la movilidad de políticas (Temenos \& McCann, 2012). En su diseño tuvieron particular incidencia las trayectorias y mundos sociales de pertenencia de estos actores: mientras que los funcionarios con experiencia en el mundo corporativo entablaron -mediante un lenguaje en común- un diálogo con el sector empresarial; los funcionarios "de carrera" dieron sustento teórico a la propuesta, incorporando los supuestos de discursos y modelos ligados a distintas formaciones discursivas, tales como la noción de cluster y de economías y ciudades creativas. Así, las tradiciones de los ministerios a cargo de la política, la trayectoria de los funcionarios de estos organismos y los discursos hegemónicos que circulan en cada uno de ellos incidieron en la particular combinación de objetivos, tradiciones teóricas y modelos de gestión pública que recupera la política de distritos porteña.

Sin embargo, los arreglos locales son siempre "extralocales" en su construcción y legitimación (Temenos \& McCann, 2012), lo que torna necesario el análisis de la dimensión relacional del proceso de movilidad. Debido al proceso desigual y selectivo de los procesos de movilidad (McCann, 2011), sólo ciertas ideas y discursos se tornan hegemónicos y, así, legitiman y condicionan las formas de concebir la ciudad, el desarrollo y las formas de gestión urbana. Esto permite explicar las continuidades que mantienen los distritos con proyectos, ideas y experiencias previas elaboradas por otras gestiones en otros momentos históricos. El abordaje discursivo propuesto aquí permitió precisamente reconocer cuáles son los supuestos y premisas que dan sustento a la política de distritos y vincularlos con estos discursos que hegemonizaron la agenda pública. Retomando nuestra pregunta inicial, vemos que la política de distritos responde a un proceso de circulación más difuso, protagonizado por actores locales; en el que las referencias a experiencias internacionales -como el 22@ Barcelona- constituyen más estrategia de legitimación, que un proceso de transferencia y/o traducción (Novick, 2005; Porto de Oliveira \& Pimenta de Faria, 2017) llevado a cabo por actores vinculados al modelo de referencia.

El artículo produce aportes al campo de la movilidad de políticas, deteniéndose no sólo en la dimensión relacional de este proceso, sino atendiendo a la dimensión territorial, a cómo la política se ancla en el territorio, superando algunas de las críticas de estos trabajos (ver Jajamovich,

\footnotetext{
19 Por motivos de espacio, no podemos explayarnos en esta cuestión, sugerimos ver González Redondo (2020) para un análisis comparado de ambas iniciativas.
} 
2018). Asimismo, produce un aporte metodológico para este abordaje: el despliegue de un dispositivo ad hoc para el análisis de los discursos que resulta productivo, sobre todo para el abordaje de la dimensión relacional de estos procesos, y como complemento de otro tipo de metodologías que tendieron a analizar la circulación de políticas. Quedan para futuros trabajos profundizar en la dimensión territorial de este proceso, analizando cómo esta política se despliega desigualmente en el territorio, produciendo dinámicas y efectos específicos.

Asimismo, el artículo constituye un aporte al campo de los estudios urbanos, en la medida en que advierte los procesos multiescalares de producción de ciudad. El abordaje de la movilidad de políticas permite matizar dos miradas explicativas predominantes sobre la producción del espacio en el contexto de reestructuración neoliberal. Por un lado, aquellos enfoques que tienden a explicar los procesos urbanos en curso como una consecuencia (más o menos determinada) del nuevo rol que asumen las ciudades en el contexto de reestructuración económica, poniendo el acento explicativo en las transformaciones globales (ver Cuervo González, 2004; Duhau, 2014). Por el otro, aquellos trabajos vinculados al campo de la gestión pública que sostienen que el reescalamiento de los territorios estratégicos otorga un rol protagónico a las ciudades como nudos articuladores de los flujos de la economía global (Borja \& Castells, 1997; Pascual i Esteve \& Foxà, 1999; Sassen, 2003, 2007; AL-LAS, 2017). En este sentido, a partir del análisis de un caso concreto, desde el abordaje de la movilidad de la políticas urbanas, el artículo se esfuerza por matizar ambos enfoques, salir de la dicotomía global-local, partiendo de una concepción multiescalar del territorio, de los procesos y las políticas urbanas, y reconociendo que los procesos de reestructuración capitalista se despliegan simultáneamente en escalas múltiples e interconectadas (Brenner, 2004).

\section{Bibliografía}

AL-LAS. Un lugar en la mesa global. Los gobiernos locales como tomadores de decisiones en la agenda mundial. Ciudad de México: Gobierno de la Ciudad de México, 2017.

AMOSSY, R. Lo plausible y lo evidente: doxa, interdiscurso, tópicos. En L'argumentation dans le discours. Discours politique, littérature d 'idées. París: Nathan, 2000, p. 1-25.

ANGENOT, M. El discurso social. México D.F.: Siglo XXI, 1989.

ARQUEROS, S La política de desarrollo de la Comuna 8 de la ciudad de Buenos Aires (1996-2015). QUID 16, 2017, N¹1, p. 331-337.

BARCELO ROCA, M. 2015. El modelo 22@ Barcelona. Disponible en http://www.redbcm.com.br/ arquivos/cidadescriativas/barcelona.pdf

BOHOSLAVSKY, E., \& SOPRANO, G. (2008). Una evaluación y propuestas para el estudio del estado en Argentina. En Un Estado con rostro humano (2010). https://doi.org/10.1080/00140139.201 2.736544

BORJA, J. \& CASTELLS, M. Local y Global. La gestión de las ciudades en la era de la información. México D.F.: Santillana, 1997. 
BRENNER, N. New State Spaces. Urban governances and the rescaling of statehood. New York: Oxford University Press, 2004.

CARAVACA, I., GONZÁLEZ, G., FERNÁNDEZ, V. \& GARCÍA, A. Economía creativa en la aglomeración metropolitana de Sevilla: agentes, redes locales de colaboración y principales actuaciones. Boletín Asociación de Geógrafos Españoles, 2013, 63, p. 81-104.

CASTELLANI, A. (2016). Consintiendo al capital. Revista MAIZ, 2016, N5. Disponible en https:// www.revistamaiz.com.ar/2016/05/consintiendo-al-capital.html

CUERVO, L.M. (2004). Ciudad y globalización en América Latina: el punto de vista de los investigadores. En Amérique Latine. Les estudes de la documentation française; Jean Michel Blanquer (pp. 1-14).

CUERVO, L.M. Ciudad y territorio en América Latina. Bases para una teoría multicéntrica, heterodoxa y pluralista. Santiago de Chile: CEPAL, 2017.

DUHAU, E. (2014). "La investigación urbana y las metrópolis latinoamericanas." En Ramírez Velázquez y Pradilla Cobos (comps.) Teorías sobre la ciudad en América Latina. México: UAM

FERNÁNDEZ, V., AMIN, A. \& VIGIL, J. Repensando el desarrollo regional. Contribuciones globales para una estrategia latinoamericana. Buenos Aires: Miño y Dávila, 2008.

FLORIDA, R. The Rise of the Creative Class. New York: Basic Books, 2002.

FLORIDA, R. (2007). “La receta es: talento, tolerancia y tecnología." HiTbarcelona.

FOUCAULT, M. La arqueología del saber. Buenos Aires: Siglo XXI, 1969.

FROST-KUMPF, H. A. (1998). Cultural Districts. The Arts as a strategy for revitalizing our cities. Illinois: American for the Arts.

GENÉ, M. En torno a los profesionales de la política. Trayectorias, prácticas y destrezas en el ejercicio del poder político desde el estado. Revista Perspectivas de Políticas Públicas, 2011, Vol. 1, No1, p. 85-107.

GIORGI, G.I. (2014). Ministros y ministerios de la Nación argentina: un aporte prosopográfico para el estudio del gabinete nacional (1854-2011). Apuntes. Revista de Ciencias Sociales, 2014, Vol. 41, No74, p. 103-139.

GOICOECHEA, M.E. Distritos Creativos en el Sur de la Ciudad de Buenos Aires (2008-2015). Renovación urbana y nuevas lógicas de segregación. Vol. 1. Buenos Aires: Universidad de Buenos Aires, 2016.

GONZÁLEZ, S. Bilbao and Barcelona "in motion". How urban regeneration "models" travel and mutate in the global flows of policy tourism. Urban Studies, 2011, Vol. 48, №7, p. 1397-1418. 
GONZÁLEZ REDONDO, C. La política de distritos en el sur de la ciudad de Buenos Aires. Modelos internacionales, actores locales y territorio (2008-2019). Tesis doctoral inédita, Facultad de Ciencias Sociales, Universidad de Buenos Aires, 2019.

GONZÁLEZ REDONDO, C. El vaciamiento que antecede la renovación. Reflexiones a partir del proyecto del distrito gubernamental en el sur de la ciudad de Buenos Aires. Territorios, 2020, No41, p. 1-22.

HOYT, L. Importing ideas: The transnational transfer of urban revitalization policy. International Journal of Public Administration, 2006, Vol. 29, Nº1-3, p. 221-243.

HYSLOP, D. Culture, Regeneration and Community. International Journal of Community Research and Engagement, 2012, N5, p. 152-65.

JAJAMOVICH, G. Puerto Madero en movimiento. Un abordaje a partir de la circulación de la Corporación Antiguo Puerto Madero (1989-2017). Buenos Aires: Teseo, 2018.

LAZZERETTI, L. El distrito cultural. Mediterráneo económico, 2008, №13, p. 327-351.

MAINGUENEAU, D. El ethos y la voz de lo escrito. Versión. Estudios de Comunicación y Política, 1996, N6, p. 79-92.

MALDIDIER, D. (1990). La inquietud del discurso. Un trayecto en la historia del análisis del discurso. El trabajo de Michel Pêcheux. Signo \& Seña, 1990, №1, p. 201-213.

MCCANN, E. Urban policy mobilities and global circuits of knowledge: Toward a research agenda. Annals of the Association of American Geographers, 2011, Vol. 101, №1, p. 107-130.

MCCANN, E. \& WARD, K. Relationality/territoriality: Toward a conceptualization of cities in the world. Geoforum, 2010, Vol. 41, №2, p. 175-184.

MENAZZI, L. ¿Una comunidad profesional de la obra pública? Empresarios, ingenieros y funcionarios durante la última dictadura (1976-1981). Revista Perspectivas de Políticas Públicas, 2018, Vol. 8, No15, p. 13-40.

MONTERO, A.S. El análisis francés del discurso y el abordaje de las voces ajenas: interdiscurso, polifonía, heterogeneidad y topos. En M. Canales (Comp.). Escucha de la escucha. Análisis e interpretación en la investigación cualitativa. Santiago de Chile: LOM-FACSO, 2014, p. 247-273.

MORRESI, S.D. \& VOMMARO, G. Saber lo que se hace: expertos y política en Argentina. Buenos Aires: Prometeo, 2011.

NOVICK, A. Planes versus proyectos: algunos problemas constitutivos del urbanismo moderno. Arquitextos, 2005, Vol. 5, №57, p. 1-8. 
NOVICK, A. La ciudad, el urbanismo y los intercambios internacionales. Notas para la discusión. Revista Iberoamericana de Urbanismo, 2009, №1, p. 4-13.

OSZLAK, O. \& O'DONNELL, G. Estado y políticas estatales en América Latina: hacia una estrategia de investigación. Redes, 1981, Vol. 2, Nº4, p. 98-128.

PASCUAL I ESTEVE, J.M \& FOXÀ, M.D.F. La estrategia de las ciudades. Los Planes Estratégicos como instrumento: Métodos, técnicas y buenas prácticas. Barcelona: Diputació de Barcelona, Oficina Tècnica de Cooperació, 1999

PECK, J. \& THEODORE, N. Mobilizing policy: Models, methods, and mutations. Geoforum, 2010, Vol. $41, N^{\circ} 2$, p. 169-174.

PORTER, M. The competitive advantage of nations. New York: The Free Press, 1990.

PORTER, M. The competitive advantage of the inner city. Harvard Bussiness Review, 1995. Disponible en: https://hbr.org/1995/05/the-competitive-advantage-of-the-inner-city\#

PORTER, M. Clusters and the new economic competition. Harvard Business Review, 1998. Disponible en https://hbr.org/1998/11/clusters-and-the-new-economics-of-competition

PORTO de OLIVEIRA, O. \& PIMENTA de FARIA, C.A. Policy Transfer, Diffusion and Circulation. Novos Estudos, 2017, Nº107, p. 13-32.

RIFFO, L. 50 años del ILPES: evolución de los marcos conceptuales sobre desarrollo territorial. Santiago de Chile: ILPES-CEPAL, 2013.

SANTOS, M. La naturaleza del espacio: técnica y tiempo, razón y emoción. Barcelona: Editorial Ariel, 1996.

SASSEN, S. Localizando ciudades en circuitos globales. EURE, 2003, Vol. 29, №88, p. 5-27.

SASSEN, S. El reposicionamiento de las ciudades y regiones urbanas en una economía global: ampliando las opciones de políticas y gobernanza. EURE, 2007, Vol. 33, №100, p. 9-34.

SOCOLOFF, I., COLOMBO, A., KITAY, I., MALTZ, H., RULLANSKY, I, SEIA, G., STIBERMAN, L. \& URDAMPILLET, M. Gobernar Buenos Aires. Un estudio sobre las racionalidades políticas en torno al desarrollo local a partir del caso del Distrito Tecnológico de Parque Patricios (2008-2012). VII Jornadas de Sociología UNLP, 2012, p. 1-18.

TEMENOS, C. \& MCCANN, E. The local politics of policy mobility: Learning, persuasion, and the production of a municipal sustainability fix. Environment and Planning A, 2012, Vol. 44, Nº, p. 1389-1406. 
VAINER, C. Pátria, empresa e mercadoria. Notas sobre a estratégia discursiva do Planejamento Estratégico Urbano. En Arantes, Vainer y Maricato (Eds.). A cidade do pensamento único. Río de Janeiro: Editora Vozes, 2000.

VERÓN, E. La semiosis social. Fragmentos de una teoría de la discursividad. Barcelona: Gedisa, 1988.

VOMMARO, G. Los partidos y sus mundos sociales de pertenencia: repertorios de acción, moralidad y jerarquías culturales en la vida política. En G. Vommaro \& M. Gené (Coords.). La vida social del mundo político. Investigaciones recientes en sociología política. Los Polvorines: UNGS, 2017.

VOMMARO, G., \& GENÉ, M. La vida social del mundo político. Investigaciones recientes en sociología política. Los Polvorines: UNGS, 2017.

VOMMARO, G. \& MORRESI, S. D. Unidos y diversificados: la construcción del partido PRO en la CABA. Revista SAAP, 2014, Vol. 8, N², p. 1666-7883.

\section{Otros documentos}

CABRERA, F. Buenos Aires, talento y creatividad. Diario La Nación. 26 de octubre de 2012. Disponible en http://www.lanacion.com.ar/1520757-buenos-aires-talento-y-creatividad.

GCBA (2009). Modelo Territorial 2010-2060.

GCBA (2009b) Manual Invertir en Buenos Aires.

GCBA (2017). Distrito de las Artes.

LCABS (2008) Fundamentos del proyecto de ley del distrito tecnológico, expediente No24.506/2008.

Revista del Consejo Profesional de Ciencias Económicas de la Ciudad Autónoma de Buenos Aires, Año V, Número 25, Noviembre de 2012, Buenos Aires. 